\title{
Validation of a Monte Carlo simulation of the Philips Allegro/GEMINI PET systems using GATE
}

\author{
F Lamare, A Turzo, Y Bizais, C Cheze Le Rest and D Visvikis \\ U650 INSERM, Laboratoire du Traitement de l'information medicale (LaTIM), CHU Morvan, \\ Université de Bretagne Occidentale, Brest, 29609, France \\ E-mail: Frederic.Lamare@univ-brest.fr
}

Received 8 April 2005, in final form 6 December 2005

Published 1 February 2006

Online at stacks.iop.org/PMB/51/943

\begin{abstract}
A newly developed simulation toolkit, GATE (Geant4 Application for Tomographic Emission), was used to develop a Monte Carlo simulation of a fully three-dimensional (3D) clinical PET scanner. The Philips Allegro/GEMINI PET systems were simulated in order to (a) allow a detailed study of the parameters affecting the system's performance under various imaging conditions, (b) study the optimization and quantitative accuracy of emission acquisition protocols for dynamic and static imaging, and (c) further validate the potential of GATE for the simulation of clinical PET systems. A model of the detection system and its geometry was developed. The accuracy of the developed detection model was tested through the comparison of simulated and measured results obtained with the Allegro/GEMINI systems for a number of NEMA NU2-2001 performance protocols including spatial resolution, sensitivity and scatter fraction. In addition, an approximate model of the system's dead time at the level of detected single events and coincidences was developed in an attempt to simulate the count rate related performance characteristics of the scanner. The developed dead-time model was assessed under different imaging conditions using the count rate loss and noise equivalent count rates performance protocols of standard and modified NEMA NU22001 (whole body imaging conditions) and NEMA NU2-1994 (brain imaging conditions) comparing simulated with experimental measurements obtained with the Allegro/GEMINI PET systems. Finally, a reconstructed image quality protocol was used to assess the overall performance of the developed model. An agreement of $<3 \%$ was obtained in scatter fraction, with a difference between $4 \%$ and $10 \%$ in the true and random coincidence count rates respectively, throughout a range of activity concentrations and under various imaging conditions, resulting in $<8 \%$ differences between simulated and measured noise equivalent count rates performance. Finally, the image quality validation study revealed a good agreement in signal-to-noise ratio and contrast recovery coefficients for a number of different volume spheres and two different (clinical
\end{abstract}


level based) tumour-to-background ratios. In conclusion, these results support the accurate modelling of the Philips Allegro/GEMINI PET systems using GATE in combination with a dead-time model for the signal flow description, which leads to an agreement of $<10 \%$ in coincidence count rates under different imaging conditions and clinically relevant activity concentration levels.

\section{Introduction}

Monte Carlo (MC) simulation studies are today an essential tool in a number of applications concerning the evolving area of emission tomography. Such applications for both single photon emission tomography (SPECT) and positron emission tomography (PET) include, among others, optimization of system design for new scanners, study of factors affecting image quality, validation of correction methodologies for effects such as scatter, attenuation and partial volume, as well as development of new reconstruction algorithms. One of the issues limiting the use of MC simulations has been long execution times. However, the advancement in individual system computing power in combination with a widespread employment of computer clusters limits such issues today only to specific emission tomography applications.

A number of different Monte Carlo simulation codes are available today, comprising different levels of adaptation and associated levels of easiness of use for emission tomography specific applications (Buvat et al 2002). The advantages of MC codes modelling particle transportation for high energy physics experiments (Agostinelli et al 2003) or dosimetry applications (Briesmeister et al 1993, Bielajew et al 1994) include exhaustive testing of the different code components as well as the experience of a wide variety of users. However, such codes do not easily facilitate the description of emission tomography systems and associated data flow under a single platform. The alternative involves the use of simulation codes developed specifically for the description of emission tomography systems (for a review, see Buvat et al (2002)). One such code is GATE (Geant4 Application for Tomographic Emission) developed by the OpenGATE collaboration (Jan et al 2004). GATE is a Monte Carlo simulator based on Geant 4 libraries, providing a scripting interface with a number of advantages for the simulation of SPECT and PET systems, including the description of source decay phenomena, moving detector components and time management (Jan et al 2004). Since the code is based on Geant4, it profits from the validation of the underlying physics components including testing from a very large scientific community. At the same time, the scripting interface provides a convenient platform for most of the users to create their own simulations of emission tomography experiments and complicated emission tomography system designs.

Several validation studies of GATE for the simulation of different clinical and small animal systems have recently been referenced in the literature. A comprehensive list exists in the recently published OpenGATE collaboration paper (Jan et al 2004). The main objectives of our study were the development of an accurate model of the Philips Allegro PET system using GATE and its validation based on system performance measurements. This is, to our knowledge, the first time that a model of this clinical PET scanner has been developed. This system is also used as the PET component in the GEMINI PET/CT scanner, and therefore the model is directly applicable to the combined PET/CT system. We have developed this model for a number of different purposes, including among them the optimization of acquisition protocols governing static and dynamic imaging regimes as well as the prediction of the impact of specific future system software upgrades. The developed model covers basic detector design and geometry as well as gantry and bed components. In addition, a dead-time 
model was developed in order to allow us to predict the count rate performance of the system, particularly under clinical imaging conditions. It should be stressed at this point that the purpose of the dead-time model developed has not been to account for the effects introduced by each individual readout electronics component. In turn the objective has been to obtain an approximate model, within current GATE capabilities, that performs well under variable imaging conditions and a wide range of count rates (largely encompassing those attained by current clinical imaging protocols) in terms of both singles and coincidences. Performance parameters, such as spatial resolution, scatter fraction and sensitivity described by the NEMA NU2-2001 (NEMA standards publication NU2-2001, Daube-Witherspoon et al (2002)) were used to assess the accuracy of the developed geometry model. On the other hand, the accuracy of the combined scanner geometry and dead-time model was assessed using count rate loss procedures under variable imaging conditions, including NEMA NU2-1994 (brain imaging) as well as standard and modified NEMA NU2-2001 (whole body imaging) protocols. Finally, an image quality test was undertaken in order to provide a measure of the overall model accuracy in the final reconstructed images. The validation step involved direct comparison of the simulated results for each of the procedures with measurements.

\section{Materials and method}

\subsection{Simulation software}

The use of GATE facilitates the description of the different components necessary for the accurate modelling of a PET system, starting from the geometric configuration up to the creation of a processing chain for the detected events. Specific geometries can be modelled by combining a number of base structures such as spheres, squares, cylinders and trapezoids. On the other hand, analytical phantoms can also be defined through the use of these base structures, while voxelized sources can be equally employed in order to represent realistic patient imaging conditions. The physics processes are based on Geant4 libraries including the modelling of radioactive source decays and interactions for standard and low energies (Agostinelli et al 2003). In addition, Geant4 material libraries in combination with userdefined materials tables are used to cover all of the object compositions necessary for the modelling of a system.

\subsection{Scanner geometry}

The Phillips Allegro PET scanner consists of one detector ring of 28 flat blocks (Surti et al 2004). Each block is made of 22 by 29 GSO crystals (638 crystals per block). Each crystal element has a surface area of $4 \times 6 \mathrm{~mm}^{2}$ with $20 \mathrm{~mm}$ in thickness. A light guide used to couple the crystal blocks with hexagonal photomultiplier tubes (PMTs) is also included in the model as a photon scattering medium. The detector arrangement results in an axial field of view (FOV) of $18 \mathrm{~cm}$, with a ring diameter of $86.4 \mathrm{~cm}$ at the surface of the detectors and a radial FOV (patient port) of $56 \mathrm{~cm}$. The lead rings $(2.86 \mathrm{~cm}$ in thickness) placed on either side of the detector ring to minimize the effects of activity from outside the FOV and the scanning bed were also included in the model. In the case of the GEMINI the PET part has shortened end shields compared to the standard Allegro system resulting in a patient port of $63 \mathrm{~cm}$ (Lodge et al 2004). A second model was used for the PET part of the GEMINI including accordingly shortened end shields. The digital model of the overall simulated Allegro geometry is shown in figure 1 . 


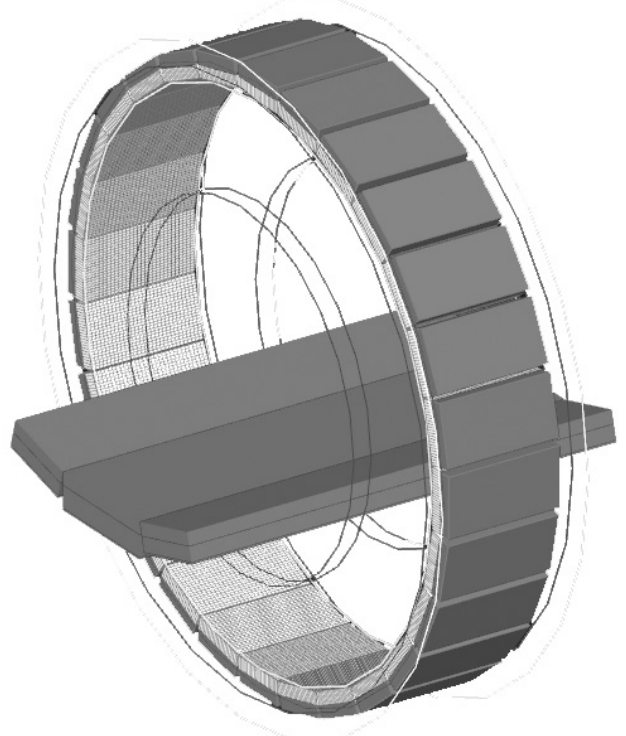

Figure 1. Graphical representation of the Allegro PET scanner model simulated using GATE, showing the crystal modules and the light guide as well as the lead shields placed on either side of the detector ring.

(This figure is in colour only in the electronic version)

\subsection{Signal flow}

The scanner is divided into 28 trigger channels. The front end dead time due to the light decay in the crystal affects every single event irrespective of whether it is part of a valid coincidence or not. The pulses from GSO have a decay time of $60 \mathrm{~ns}$, needing $\sim 200 \mathrm{~ns}$ of integration if all the scintillation light is to be collected. In order to minimize pulse pile-up effects, pulse shaping is performed by the preamplifiers to shorten the pulses. Consequently, each of the 28 trigger channels is associated with a dead time of $80 \mathrm{~ns}$, which has been modelled using the non-paralysable model (Knoll 2000). An estimation of the magnitude and the model for the applied dead time on the level of single events has been provided by the manufacturer (Kolthammer 2004). At the level of detected single events an energy window of between 210 and $665 \mathrm{keV}$ is considered.

Each trigger signal passes through a constant fraction discriminator to obtain trigger timing information. These signals are subsequently checked for coincidence events. Once a coincidence is detected (coincidence time window $2 \tau$ of $7.5 \mathrm{~ns}$ ), the digitized PMT signals within the two blocks in coincidence are read up, followed by calculations of the local energy and local position centroid (Karp et al 1986, Surti et al 2004). After a coincidence event is detected, the signals from the PMTs corresponding to the associated singles trigger channels are integrated. The PMTs are continuously digitized $(50 \mathrm{MHz})$ resulting to a digital coincidence circuit dead time which was modelled using also the non-paralysable model and an associated dead time of $80 \mathrm{~ns}$. Coincidences are allowed between each of the 28 blocks (forming the complete detector gantry) and the opposing 13 blocks. Single events with energy between $410 \mathrm{keV}$ and $665 \mathrm{keV}$ are considered in the formation of coincidence events.

No explicit modelling of pulse pile-up has been included since PMT pulses for individual photon interactions, in addition to overlapping triggering channels, will have to be considered 


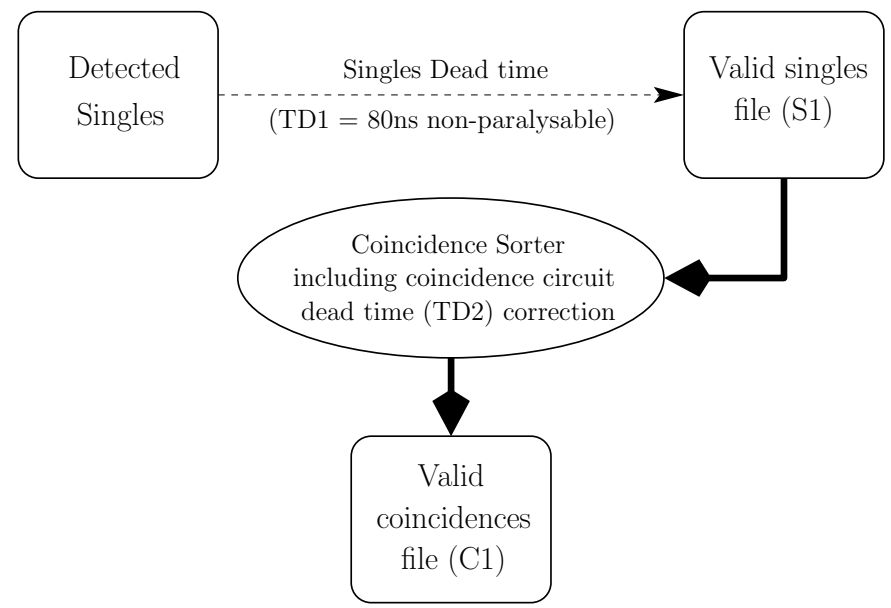

Figure 2. Graphical representation of the dead-time model implemented for the Philips Allegro/GEMINI system's signal flow.

for accurate modelling of such effects; a feature that is not currently implemented in GATE. Such effects are expected to be more significant at high count rates and they can lead to not simply count rates losses but also contrast and image resolution degradation. However, positioning errors from pulse pile-up can, in turn, be reduced by the use of local position centroid algorithms implemented in these systems (Karp et al 1986, Surti et al 2004). The potential of introducing spatial resolution changes and contrast errors at clinical activity levels as a result of omitting the explicit modelling of pulse pile-up effects have been investigated using the spatial resolution and image quality phantoms (see sections 2.4.1 and 2.4.4). On the other hand, different geometry phantoms have been used to assess the accuracy and the limitations of the developed dead-time model on the level of single events under different count rate levels and different imaging conditions (see section 2.4.2).

Multiple raw data format outputs are available for a PET scanner simulation within GATE (Jan et al 2004). In our case, we have used either the ASCII or ROOT (Brun and Rademakers 1997) output providing a list mode file of the detected single events including energy deposited and coordinates of detection within the modelled scanner geometry for each single event. The dead-time modelling, accounting for the effects outlined above, was implemented in two separate steps (see also figure 2).

As a first step, the non-paralysable dead time (TD1) of $80 \mathrm{~ns}$ is applied to the singles output (considering all detected singles with energy between 210 and $665 \mathrm{keV}$ ). A new singles file $(\mathrm{S} 1)$ is produced containing the events not rejected by the application of the dead-time model. Only the singles whose energy is within $410-665 \mathrm{keV}$ are finally stored in this file.

The singles included in the S1 file, produced in the first step, are subsequently used to create a list mode file $(\mathrm{C} 1)$ containing all the valid coincidences. Coincidences are allowed between singles registered within the coincidence time window of $7.5 \mathrm{~ns}$ in each of the detector blocks and the 13 opposing blocks. The non-paralysable dead time (TD2) of $80 \mathrm{~ns}$ associated with the digital coincidence circuit is also applied at this stage. The accuracy of the magnitude and the model of the dead time considered at the level of coincidences has been tested under different count rate levels and different imaging conditions (see section 2.4.2).

Finally, signal flow effects such as electronics bandwidth limits and transfer to disk effects have not been included in the dead-time model which could affect its performance, particularly at high count rates. 
Following the formation of the coincidences file, the number of true, random and scatter coincidences can be counted. In the GATE singles output, each line corresponds to a detected photon. For each photon, along with its origin (annihilation) and detection coordinates, there is a flag specifying if and how many Compton interactions have occurred during the tracking of the photon. This information is used in the classification of scatter coincidences. The information on the annihilation coordinates for each of the detected single events is subsequently used to determine which of the accepted coincidences can be classified as randoms.

\subsection{Validation studies}

The National Electrical Manufacturers Association (NEMA) performance tests are widely accepted as the measurements of standard for the assessment of individual PET system's performance and inter-scanner comparisons. We have chosen to use both the NEMA NU22001 guidelines (Daube-Witherspoon et al 2002) and NEMA NU2-1994 guidelines to validate our developed GATE model of the Allegro/GEMINI PET systems. The tests outlined in these procedures have been particularly devised in order to provide a comprehensive description of the performance of a PET system in whole body (NEMA NU2-2001) and brain (NEMA NU2-1994) imaging. Parameters including spatial resolution, scatter fraction and sensitivity were simulated according to the NEMA NU2-2001 protocols, while both NEMA NU2-2001 and NU2-1994 guidelines were used to simulate count rate losses. The NEMA NU2-2001 consists of a solid polyethylene cylinder (diameter: $19.5 \mathrm{~cm}$, height: $70 \mathrm{~cm}$ ) and a $70 \mathrm{~cm}$ long line source placed at $4.5 \mathrm{~mm}$ radial offset from the centre of the cylinder, whereas the NEMA NU2-1994 is a circular cylinder composed of polymethyl methacrylate (PMMA, inside diameter: $19.5 \mathrm{~cm}$, interior length: $19 \mathrm{~cm}$ ). Finally, overall image quality was simulated using the NEMA NU2-1994 cylinder and compared to measurements (Visvikis et al 2004). The simulated cylinders used in the validation phase are shown in figure 3.

2.4.1. Spatial resolution. To assess the spatial resolution of our modelled scanner, the simulated $1 \mathrm{~mm}$ diameter sphere was uniformly filled with ${ }^{18} \mathrm{~F}$ and placed at six different positions in the active field of view. More specifically, the point source was placed at two different axial locations; namely at the centre and at one-fourth of the axial FOV. For each of these two axial locations, three different positions were also covered, at $1 \mathrm{~cm}$ and $10 \mathrm{~cm}$ vertically from the centre as well as at $10 \mathrm{~cm}$ horizontally from the centre. In addition, measurements following the NEMA protocol for spatial resolution were performed using the local Allegro system within Brest University Hospital.

The simulated and measured data were reconstructed using Fourier rebinning (FORE) followed by two-dimensional (2D) filtered backprojection (ramp filter) as used to obtain the literature measurements (Surti et al 2004, Lodge et al 2004) that we are using for comparison with the simulated results. The spatial resolution on each of the six different positions considered was subsequently calculated by determining the full-width at half-maximum (FWHM) of the resulting point spread function obtained by interpolation between adjacent pixels in the radial, tangential and axial profiles.

2.4.2. Coincidence count rates, scatter fraction, noise equivalent count rates. Different phantom geometries simulating different imaging conditions were used in order to evaluate the accuracy and potential shortcomings of our dead-time model. Firstly, the count rate measurements based on the NEMA NU2-2001 protocol were simulated by filling the $70 \mathrm{~cm}$ long line source uniformly with ${ }^{18} \mathrm{~F}$. In addition to the standard NEMA NU2-2001 cylinder 

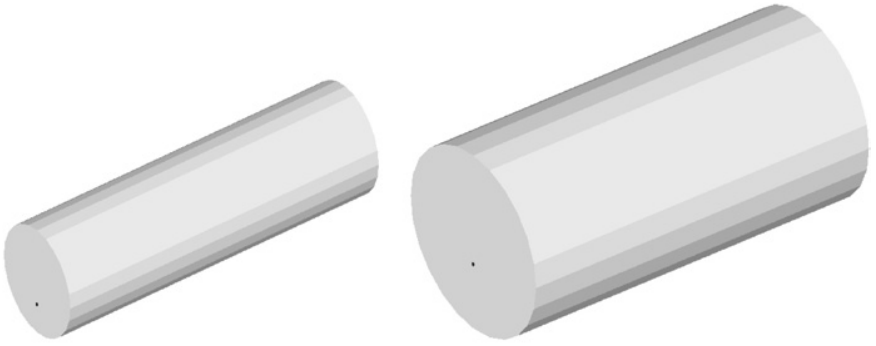

(a)
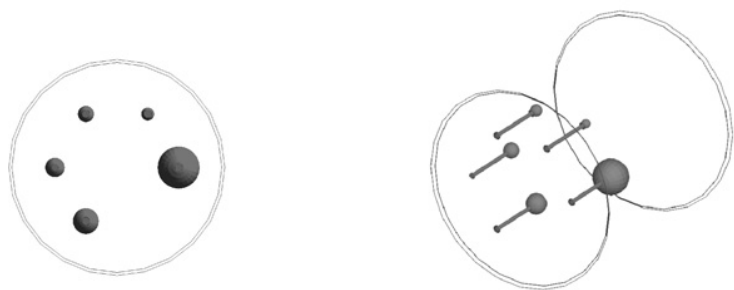

(b)

Figure 3. View of the simulated phantom geometry of (a) NEMA NU2-2001, standard $20 \mathrm{~cm}$ diameter and modified $35 \mathrm{~cm}$ diameter (used in the count rate loss and scatter fraction measurements), and (b) NEMA NU2-1994 uniform cylinder (used in the count rate loss and scatter fraction measurements) including a number of spheres (used in the image quality measurements).
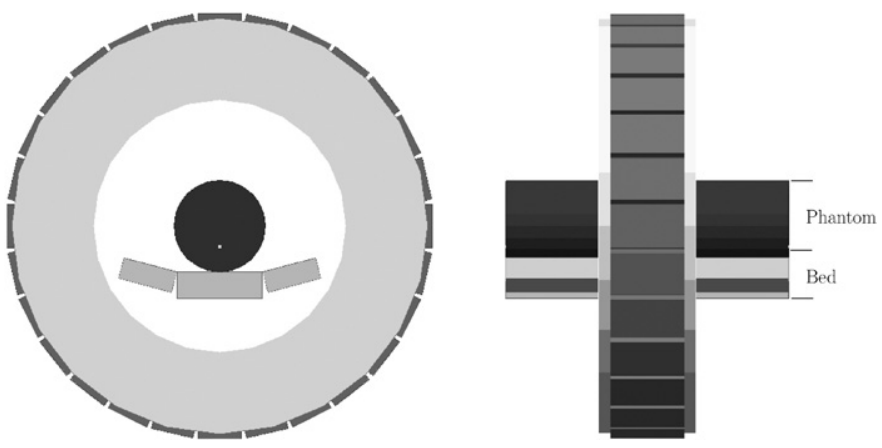

Figure 4. Position of the NEMA NU2-2001 phantom (including the $70 \mathrm{~cm}$ long line source insert) within the simulated scanner geometry for the count rate performance assessment.

diameter $(20 \mathrm{~cm})$, a second phantom with a diameter of $35 \mathrm{~cm}$ was used in order to simulate larger patients imaging conditions (Surti et al 2003). The length of the cylinder was kept unchanged at $70 \mathrm{~cm}$. Finally, the NEMA NU2-1994 was simulated uniformly filled with a solution of water and ${ }^{18} \mathrm{~F}$ in order to investigate the performance of our signal flow model under brain imaging conditions. All cylindrical phantoms were placed at the centre of the axial and transaxial fields of view of the modelled scanner (see figure 4). Progressively larger amounts of activity were simulated in the source (line source for the NEMA NU2-2001 phantoms, entire cylinder for the NEMA NU2-1994 phantom) in order to recover the count rate behaviour of the system as a function of activity present in the phantom. The recorded singles ASCII files for each of the activity levels simulated were processed for dead-time effects using the scheme described in section 2.3 and demonstrated in figure 2. This was to facilitate both a singles and 
a coincidence count rates validation as a function of activity for the developed model. The comparison between simulated and measured singles was only carried out for the standard NEMA NU2-2001 and NU2-1994 phantoms, where measured singles data were available. Using the simulated count rates the scatter fraction was calculated as

$$
\mathrm{SF}=\frac{S}{S+T}
$$

where $S$ and $T$ are the number of true and scattered coincidences identified as described in section 2.3. In addition, the noise equivalent count (NEC) rates as a function of activity were calculated from the simulated count rates and compared to measurements using the following expression,

$$
\mathrm{NEC}=\frac{T^{2}}{T+S+k R}
$$

where $T, S$ and $R$ are the number of trues, scattered and random coincidences, respectively. As specified in the NEMA protocol a $24 \mathrm{~cm}$ diameter circular region centred on the standard $20 \mathrm{~cm}$ diameter phantom was used during the estimation of the simulated count rates. In the case of the larger $35 \mathrm{~cm}$ diameter phantom, a circular region of $39 \mathrm{~cm}$ was used (Surti et al 2003). Any detected coincidences lying outside this region were not considered. A $k$ of 1 was used denoting a noiseless randoms correction used during the direct measurements of NEC rates (Surti et al 2004, Lodge 2004).

2.4.3. Sensitivity. The NEMA NU2-2001 sensitivity phantom consists of a $700 \mathrm{~mm}$ long line source and five concentric aluminium tubes placed around the line source. The aluminium tubes are also $700 \mathrm{~mm}$ long and can be stacked one inside the other. The phantom is placed at the centre of the scanner, including initially the line source and the first aluminium tube, increasing progressively the attenuation through the use of increasing number of metal sleeves around the source.

The simulated line source was uniformly filled with ${ }^{18} \mathrm{~F}$. The activity concentration was chosen so that the single events counting losses are less than $1 \%$ and the random event rate is less than $5 \%$ of the trues rate as specified by the NEMA protocol. The simulation time is chosen to ensure that at least 10000 true coincidences per slice are collected.

Since the data collection is performed in $3 \mathrm{D}$, single slice rebinning is used to assign coincidence events from oblique LORs to the transverse slice where the oblique LOR crosses the scanner axis. Ten different simulations were performed; one simulation for each aluminium sleeve at the centre and at $10 \mathrm{~cm}$ from the centre of the transaxial FOV. The natural logarithm of the true coincidences count rate was plotted as a function of the overall aluminium thickness, for the two transaxial positions. A linear regression was used to fit the simulated results and compute the absolute sensitivity of the scanner in the absence of attenuating material. The results for the smallest aluminium tube at the central transaxial position were used to plot the sensitivity profile.

2.4.4. Image quality. In order to assess the overall quality of our model, accounting for the interactions between parameters that the different performance tasks are assessing, we have included an image quality assessment task. The phantom used is shown in figure 3(b). It consists of the NEMA NU2-1994 cylinder $(20 \mathrm{~cm}$ and $21.4 \mathrm{~cm}$ in diameter and height, respectively) uniformly filled with ${ }^{18} \mathrm{~F}$ and five fillable hollow glass spheres of variable diameters $(10,13,17,22$ and $37 \mathrm{~mm}$ internal diameter). Measurements were carried out using an emission scan time of 3 min over a single bed position, which is the typical time of acquisition per axial field of view used during clinical whole body studies allowing good 
contrast and quantitative accuracy (Surti et al 2004, Visvikis et al 2004). Two different sphere-to-background ratios of $8: 1$ and 4:1 were measured and simulated using the same activity concentrations $\left(7.4 \mathrm{kBq} \mathrm{ml}^{-1}\right.$ in the background uniform part).

The simulated list mode files corrected for dead-time effects were rebinned to the Philips proprietary sinogram format in order to allow their reconstruction using the clinical scanner software. Only the prompt coincidences were considered from the simulated datasets as the direct online randoms subtraction was used during the measurements. In general, direct online randoms subtraction is an efficient and accurate method of correction for the presence of random coincidences. Following such an operation noise levels in the reconstructed images are generally higher depending on the level of activity concentration considered (Brasse et al 2005). However, such differences should not influence our comparison between simulated (randoms not included) and measured results, since firstly the influence of noise is minimal in the estimation of average activity concentrations (Brasse et al 2005) and secondly the effects on measurements of signal-to-noise ratio are kept to minimum by considering activity concentrations which result in a randoms fraction of $<7 \%$.

Measured (Visvikis et al 2004) and simulated sinograms were reconstructed using the 2.5D RAMLA algorithm with the default parameters used in routine clinical whole body image reconstructions ( 2 iterations, blob size and shape of 2.5 and 8.63 respectively). Parameters such as blob size and shape in the RAMLA algorithm can influence the noise characteristics and the level of smoothing in the reconstructed images. The values chosen have been shown to lead to higher contrast images without an associated increase in image noise (Visvikis et al 2004). The parabolic tail fitting was used for scatter correction (Surti et al 2004). The simulated and measured image quality phantoms were positioned exactly at the same place, so the same transmission image was used to correct the measured and simulated datasets for the effects of attenuation. The transmission scan was obtained using a137Cs source followed by segmented attenuation correction (Smith et al 1997, Talguen et al 2004). Subsequently, regions of interest (ROIs) were placed over the spheres in a single plane passing through their centre. The size of each of the ROIs was that of the known sphere. Finally, ten ROIs of $30 \mathrm{~mm}$ in diameter were placed on the uniform background on each of four adjacent planes. The mean count densities and standard deviations were recorded for each of the ROIs. Using the mean count densities, the percentage contrast recovery coefficient was calculated using

$$
\mathrm{CRC}=\frac{N_{i} / N_{b}-1}{A_{i} / A_{b}-1}
$$

where $N_{i}$ and $N_{b}$ are the average counts in the ROI for sphere $j$ and the background respectively, while $A_{i}$ and $A_{b}$ are the activity concentrations in the spheres and in the background respectively.

Finally, for each of the reconstructed images, a report of an image signal-to-noise was calculated as the ratio between the mean background count densities and the corresponding standard deviation.

\section{Results}

\subsection{Spatial resolution}

Table 1 contains the spatial resolution results in terms of FWHM (mm) for all six positions where point source measurements and simulations were carried out. In addition, according to the NEMA NU2-2001 protocol, table 2 contains values for the radial and tangential resolution averaged over both axial positions, for each radial position considered $(1 \mathrm{~cm}$ and $10 \mathrm{~cm})$. 
Table 1. Spatial resolution results for different axial, radial and tangential positions, for both measured and simulated point sources at corresponding locations within the FOV.

\begin{tabular}{llllll}
\hline & \multicolumn{2}{c}{ Tangential FWHM $(\mathrm{mm})$} & & \multicolumn{2}{c}{ Radial FWHM (mm) } \\
\cline { 2 - 3 } Position & Measurement & Simulation & & Measurement & Simulation \\
\hline$Y=1 \mathrm{~cm}$, axial $=0$ & 5.23 & 4.74 & & 5.64 & 4.56 \\
$Y=1 \mathrm{~cm}$, axial $=1 / 4$ & 5.30 & 4.60 & & 5.55 & 4.56 \\
$Y=10 \mathrm{~cm}$, axial $=0$ & 5.25 & 4.49 & & 5.55 & 4.79 \\
$X=10 \mathrm{~cm}$, axial $=0$ & 5.35 & 4.60 & & 5.86 & 4.89 \\
$Y=10 \mathrm{~cm}$, axial $=1 / 4$ & 5.23 & 4.32 & & 5.70 & 4.73 \\
$X=10 \mathrm{~cm}$, axial $=1 / 4$ & 5.20 & 4.57 & 6.08 & 4.98 \\
\hline
\end{tabular}

Table 2. Spatial resolution for two different radial positions $(1 \mathrm{~cm}$ and $10 \mathrm{~cm}$ from the centre of the FOV), calculated using the NEMA NU2-2001 protocols.

\begin{tabular}{lllll}
\hline & $\begin{array}{l}\text { Allegro } \\
\text { measurements } \\
\text { (Brest University } \\
\text { Fospital) }\end{array}$ & $\begin{array}{l}\text { Allegro } \\
\text { measurements } \\
\text { (Surti } \text { et al } \\
\text { 2004) }\end{array}$ & $\begin{array}{l}\text { GEMINI } \\
\text { measurements } \\
\text { (Lodge } \text { et al } \\
\text { 2004) }\end{array}$ & $\begin{array}{l}\text { GATE } \\
\text { simulations }\end{array}$ \\
\hline Radial offset $=1 \mathrm{~cm}$, transverse & 5.43 & 5.49 & 4.9 & 4.57 \\
Radial offset $=1 \mathrm{~cm}$, axial & 5.56 & 5.63 & $\mathrm{~N} / \mathrm{A}$ & 4.49 \\
Radial offset $=10 \mathrm{~cm}$, transverse tangential & 5.26 & N/A & 5.2 & 4.41 \\
Radial offset $=10 \mathrm{~cm}$, transverse radial & 5.70 & N/A & 5.7 & 4.90 \\
Radial offset $=10 \mathrm{~cm}$, transverse average & 5.48 & 5.65 & 5.45 & 4.67 \\
Radial offset $=10 \mathrm{~cm}$, axial & 7.27 & 7.45 & N/A & 6.15 \\
\hline
\end{tabular}

The spatial resolution values obtained using the simulated model are within $11-18 \%$ of our measured results or considering already published data. In addition, the simulated results are consistently demonstrating better spatial resolution in comparison to the measured values, mainly due to the absence of modelling within GATE the process of light spreading and light sharing between PMTs. No differences were seen in the values obtained using simulated data from the Allegro and the GEMINI models, something that is in broad agreement with literature results summarized in table 2 .

\subsection{Count rates}

Figure 5 shows a comparison of simulated and measured singles count rates as a function of increasing activity concentration for the $20 \mathrm{~cm}$ diameter by $70 \mathrm{~cm}$ long NEMA NU2-2001 and the NEMA NU2-1994 phantoms. The simulated results include the effects of modelled dead time (see schema in figure 2). The simulated singles count rates, covering a range from 1 to $30 \mathrm{Mcps}$, are within $6 \%$ of the measured count rates throughout a wide range of activity concentrations and different imaging conditions represented by the two phantom geometries considered.

Using the methodology described in section 2.4.2 and applying the coincidences deadtime model described in figure 2, corresponding coincidence count rates have been derived for all of the phantoms considered. The different types of simulated coincidence count rates as a function of increasing activity concentration are shown in figure 6 in comparison with count rates in the literature (Surti et al 2003, Surti 2004).

Table 3 contains a summary of measured and simulated count rates at specific activity concentrations for the standard NEMA NU2-2001 phantom geometry. This table also helps in demonstrating count rate differences between the Allegro and the GEMINI geometries 


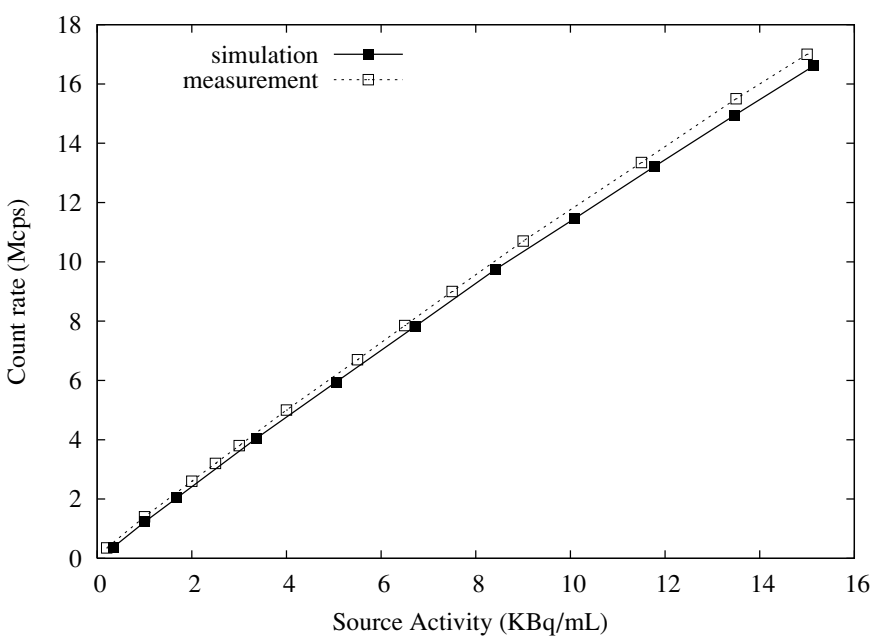

(a)

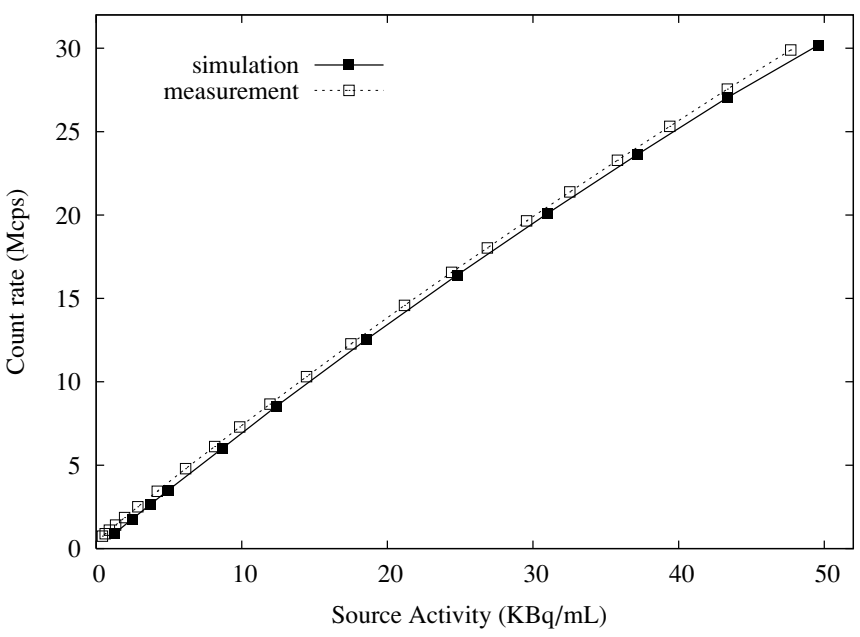

(b)

Figure 5. Simulated singles count rates (including dead-time modelling) compared with measurements (Surti et al 2004) as a function of increasing activity concentration for (a) the standard NEMA NU2-2001, and (b) NU2-1994 phantoms.

Table 3. Measured and simulated coincidences count rate performance based on the NEMA NU2-2001 protocol for the Allegro and GEMINI geometries.

\begin{tabular}{lllll}
\hline & $\begin{array}{l}\text { Measured } \\
\text { (Allegro, Surti } \\
\text { et al 2004) }\end{array}$ & Simulated & $\begin{array}{l}\text { Measured } \\
\text { (GEMINI Lodge } \\
\text { et al 2004) }\end{array}$ & Simulated \\
\hline $\begin{array}{l}\text { Activity coincidences }\left(\mathrm{kBq} \mathrm{ml}^{-1} \text { ) for peak trues }\right. \\
\text { Peak True coincidences rates }(\mathrm{kcps}) \text { [Simulated rates }\end{array}$ & 13.7 & 15.9 & 13.8 & 15.8 \\
at measured peak trues activity coincidences] & $83[83.4]$ & 86.2 & $77.1[77.8]$ & 80.6 \\
Randoms $=$ trues activity coincidences $\left(\mathrm{kBq} \mathrm{cc}^{-1}\right)$ & 11 & 10.3 & 10.8 & 9.2 \\
Randoms $=$ trues coincidences rate $(\mathrm{kcps})$ & 80 & 75.5 & 73 & 68.4 \\
\hline
\end{tabular}




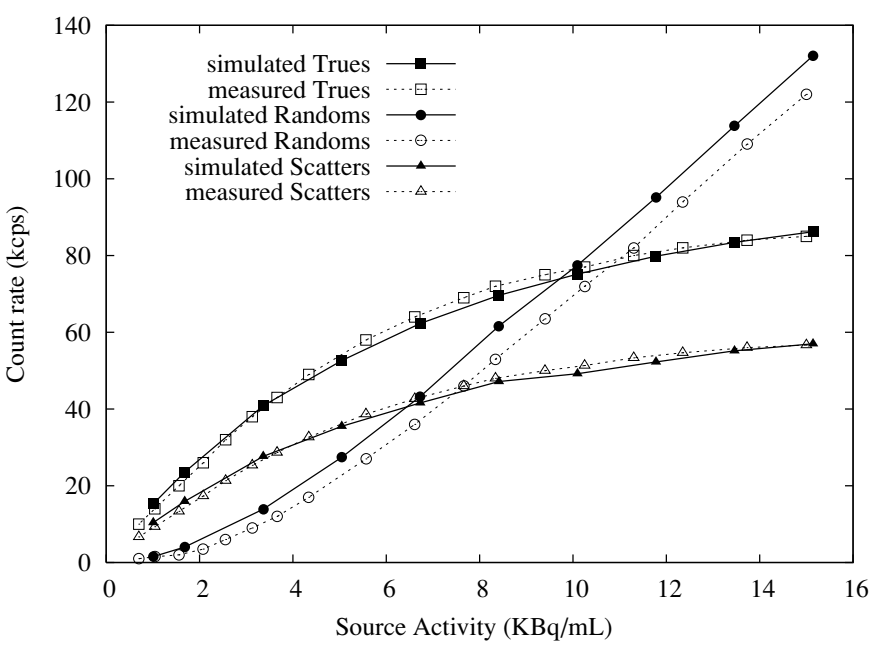

(a)

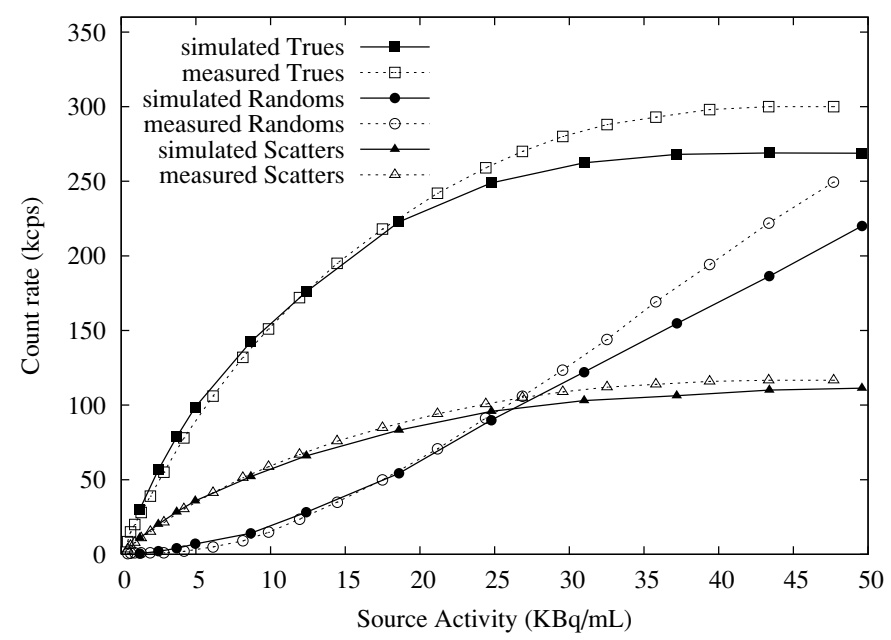

(b)

Figure 6. Simulated true, random and scatter coincidences count rates (including dead-time modelling) compared with measurements from the literature (Surti et al 2004, Surti et al 2003) as a function of increasing activity concentration for (a) the standard NEMA NU2-2001, (b) the NU2-1994, and (c) the $35 \mathrm{~cm}$ diameter NEMA NU2-2001 phantoms.

(with shortened end shields used in the case of the GEMINI). Considering low counting rates $\left(1 \mathrm{kBq} \mathrm{ml}^{-1}\right)$ as specified by the NEMA NU2-2001 protocol, a simulated scatter fraction of $40.5 \%$ and $40.9 \%$ was found for the Allegro and the GEMINI geometries in comparison to $40 \%$ obtained from measurements (Lodge et al 2004, Surti et al 2004).

In the case of the NEMA NU2-1994, a simulated peak true coincidences rate of $265 \mathrm{kcps}$ was obtained at $49.8 \mathrm{kBq} \mathrm{ml}^{-1}$, compared to a measured peak true coincidences rate of $300 \mathrm{kcps}$ at an activity concentration of $47.7 \mathrm{kBq} \mathrm{ml}^{-1}$ (figure 6(b)). For the same phantom, a simulated scatter fraction of $26.3 \%$ is also within $3 \%$ of the measured scatter fraction of $27.1 \%$. 


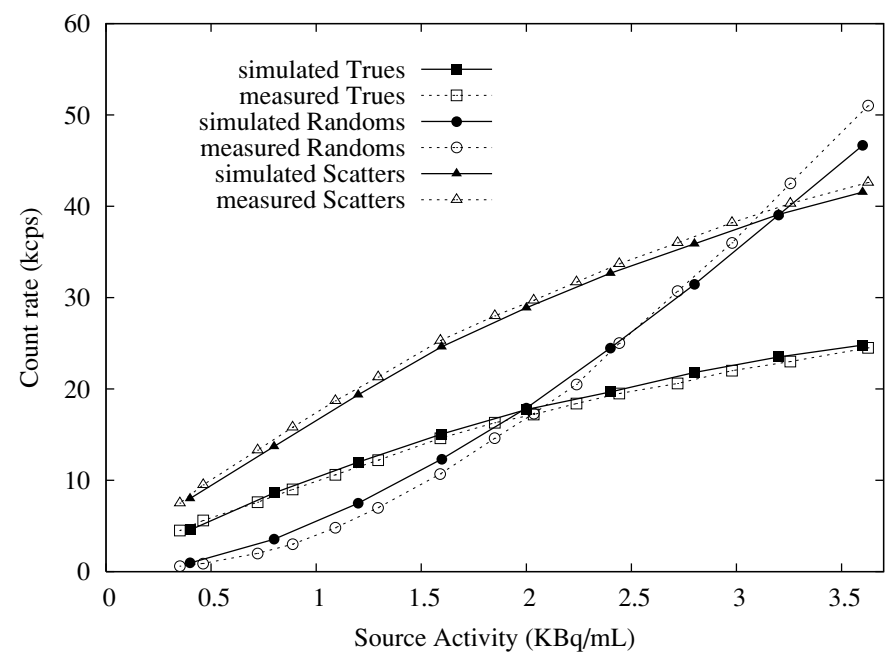

(c)

Figure 6. (Continued.)

Figure 6(c) illustrates a good agreement between all different coincidences count rates for the larger diameter NEMA NU2-2001 phantom, while a $<3 \%$ difference was seen between simulated $(61.3 \%)$ and measured $(63 \%)$ scatter fractions.

Finally, figure 7 shows the simulated noise equivalent count rates as a function of activity concentration derived using the coincidence count rates of figure 6. Considering the standard NEMA NU2-2001 phantom, peak simulated NEC rates are at $29 \mathrm{kcps}$ for $10.1 \mathrm{kBq} \mathrm{ml}^{-1}$ in comparison to a measurement of $30 \mathrm{kcps}$ at $9.25 \mathrm{kBq} \mathrm{ml}^{-1}$ (at this activity concentration the simulated NEC rates are $28.9 \mathrm{kcps}$ ). In the case of the GEMINI geometry with shortened end shields, the simulated peak NEC rates are at $26.2 \mathrm{kcps}$ for an activity concentration of $10.1 \mathrm{kBq} \mathrm{ml}^{-1}$ in comparison to a measured peak NEC rate (Lodge 2004) of $27.8 \mathrm{kcps}$ at $9.0 \mathrm{kBq} \mathrm{ml}^{-1}$ (at this activity concentration the simulated NEC rates are $26 \mathrm{kcps}$ ). Under brain imaging conditions simulated using the NEMA NU2-1994 phantom, a peak NEC of $142.9 \mathrm{kcps}$ and $152.2 \mathrm{kcps}$ was obtained at $27.8 \mathrm{kBq} \mathrm{ml}^{-1}$ and $29.6 \mathrm{kBq} \mathrm{ml}^{-1}$ for simulated and measured data respectively (figure 7(b)). Finally, in the case of the modified NEMA NU2-2001 phantom a simulated peak NEC of $5.3 \mathrm{kcps}$ at $3.2 \mathrm{kBq} \mathrm{ml}^{-1}$ compares well with a measured peak NEC of $5 \mathrm{kcps}$ at $3.3 \mathrm{kBq} \mathrm{ml}^{-1}$ (figure 7(c)).

\subsection{Sensitivity}

Table 4 shows a comparison of the simulated and measured (Surti et al 2004) absolute sensitivity for the Allegro scanner at two positions in the field of view. The simulated measurements are $<10 \%$ higher than the measured sensitivity. Furthermore, table 4 also reveals that the ratio between the sensitivity at the two positions in the field of view was practically the same between the simulated and the measured results. The axial sensitivity profile is plotted in figure $8(\mathrm{c})$, demonstrating a peak at $0.23 \mathrm{cps} \mathrm{kBq}^{-1}$ compared to a measured maximum axial sensitivity of $\sim 0.2 \mathrm{cps} \mathrm{kBq}^{-1}$ (Surti et al 2004). 


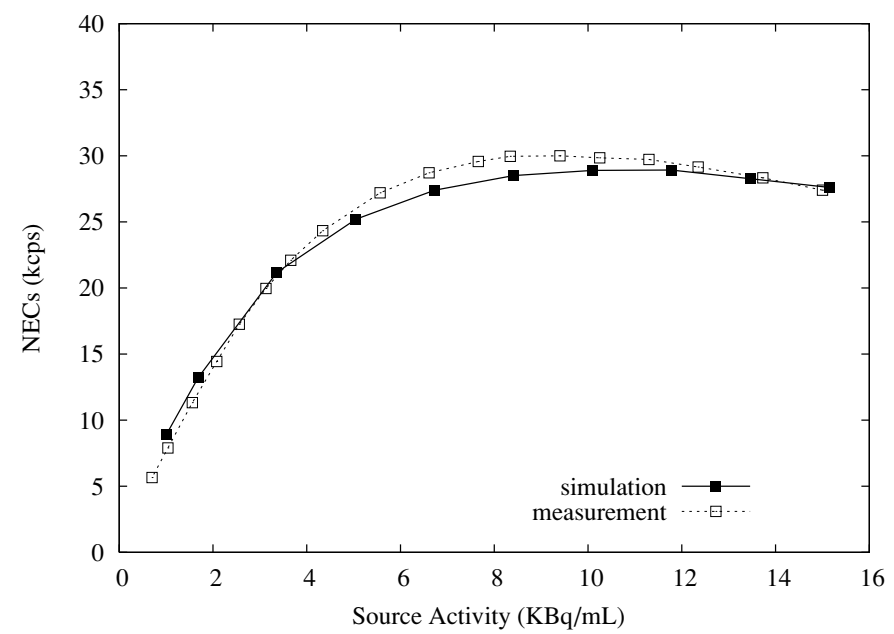

(a)

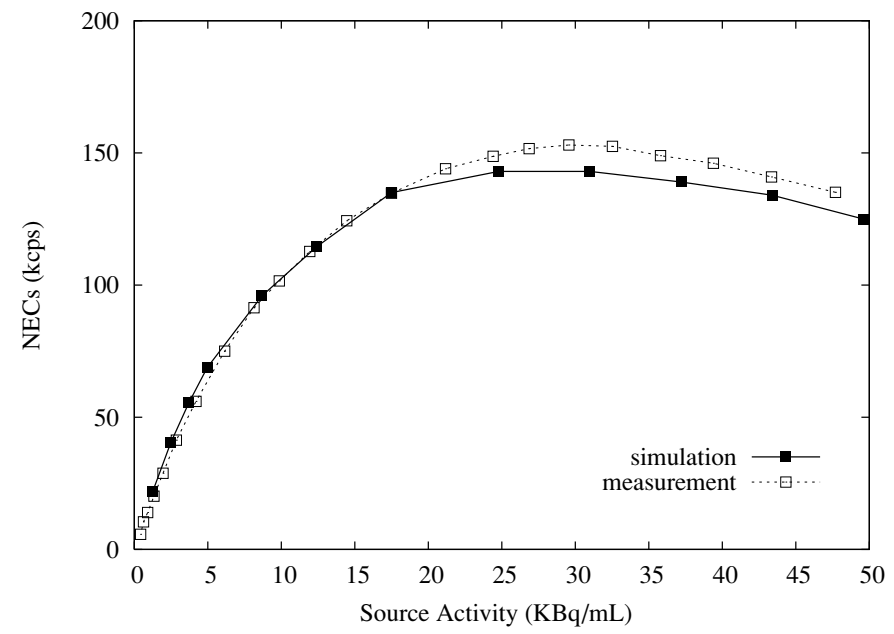

(b)

Figure 7. Measured (Surti et al 2003, 2004) and simulated NEC rate curves for (a) the standard NEMA NU2-2001, (b) the NU2-1994, and (c) the $35 \mathrm{~cm}$ diameter NEMA NU2-2001 phantoms, based on the coincidences data presented in figure 6 .

Table 4. Simulated absolute sensitivity values for the Allegro compared with measurements.

\begin{tabular}{lll}
\hline Transaxial offset position & Measured values & Simulated values \\
\hline $0 \mathrm{~cm}$ & $4.36 \mathrm{cps} \mathrm{kBq}^{-1}$ & $4.88 \mathrm{cps} \mathrm{kBq}^{-1}$ \\
$10 \mathrm{~cm}$ & $4.60 \mathrm{cps} \mathrm{kBq}^{-1}$ & $5.17 \mathrm{cps} \mathrm{kBq}^{-1}$ \\
Ratio $(0 \mathrm{~cm} / 10 \mathrm{~cm})$ & 0.948 & 0.944 \\
\hline
\end{tabular}

\subsection{Image quality}

The percentage contrast recovery coefficients (CRC) as a function of sphere size for the image quality phantom are shown in figure 9. The results for two different sphere-to-background 


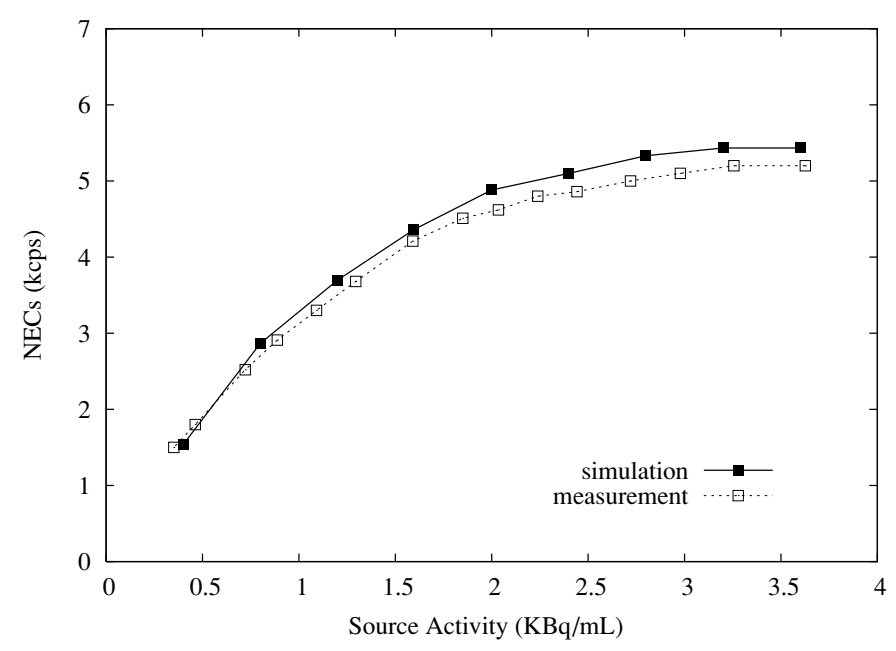

(c)

Figure 7. (Continued.)

Table 5. Measured and simulated background signal-to-noise ratio using the NEMA NU2-1994 phantom with two different sphere-to-background ratios (namely 4:1 and 8:1).

\begin{tabular}{lll}
\hline Sphere-to-background ratios & Measured values & Simulated values \\
\hline 4 to 1 & $6.89 \pm 0.78$ & $6.41 \pm 0.95$ \\
8 to 1 & $7.39 \pm 0.65$ & $7.68 \pm 0.83$ \\
\hline
\end{tabular}

ratios (4:1 and 8:1) are included. A generally good agreement is observed throughout the range of sphere sizes considered, with an average absolute difference of between $1 \%$ and $8 \%$. No significant differences were seen in terms of the agreement between simulated and measured CRCs for the two different sphere-to-background ratios. Finally, table 5 contains the average background signal-to-noise ratio and their standard deviation (considering the ten background ROIs used for each of the images). Similar signal-to-noise ratios (within the limits defined by the standard deviation) were observed for the images reconstructed using the measured and the simulated datasets, irrespective of the sphere-to-background ratio considered.

\section{Discussion}

The objectives of this work were to develop an MC model of the Philips Allegro PET scanner based on the use of GATE and validate its accuracy against experimental measurements. A modified version of this model considering the shorter end shields for the PET component of the combined GEMINI PET/CT system was also evaluated.

In addition to modelling the scanner geometry, a dead-time model was developed to account for data loss in both the singles and the coincidences level. Furthermore, tools were developed to facilitate binning of the valid coincidences to the Philips sinogram proprietary format ensuring the faithful reproduction of the image reconstruction process including the different data corrections applied prior to producing reconstructed images. The validation of the geometry model was based on a number of the current NEMA NU2-2001 standards 


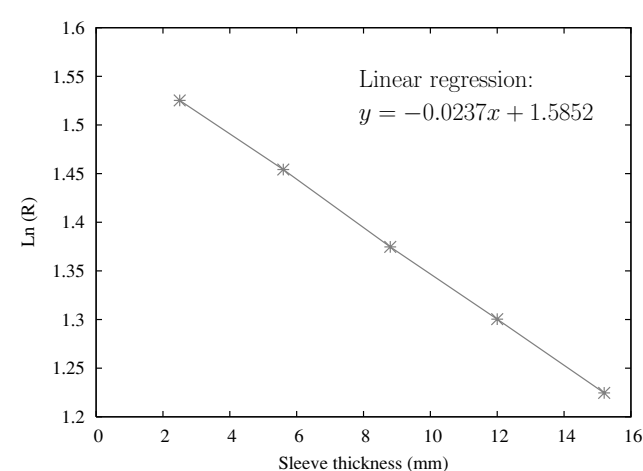

(a)

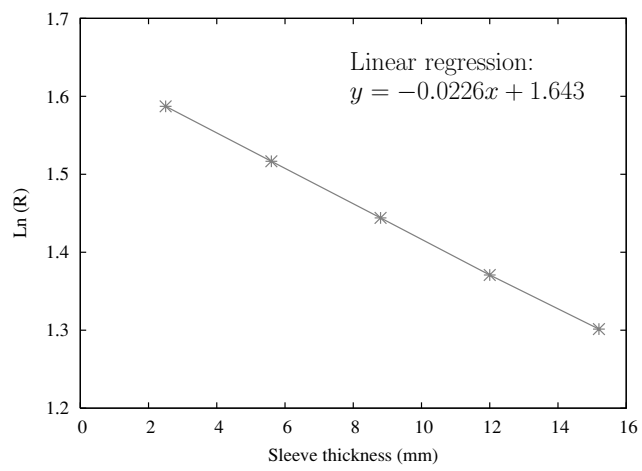

(b)

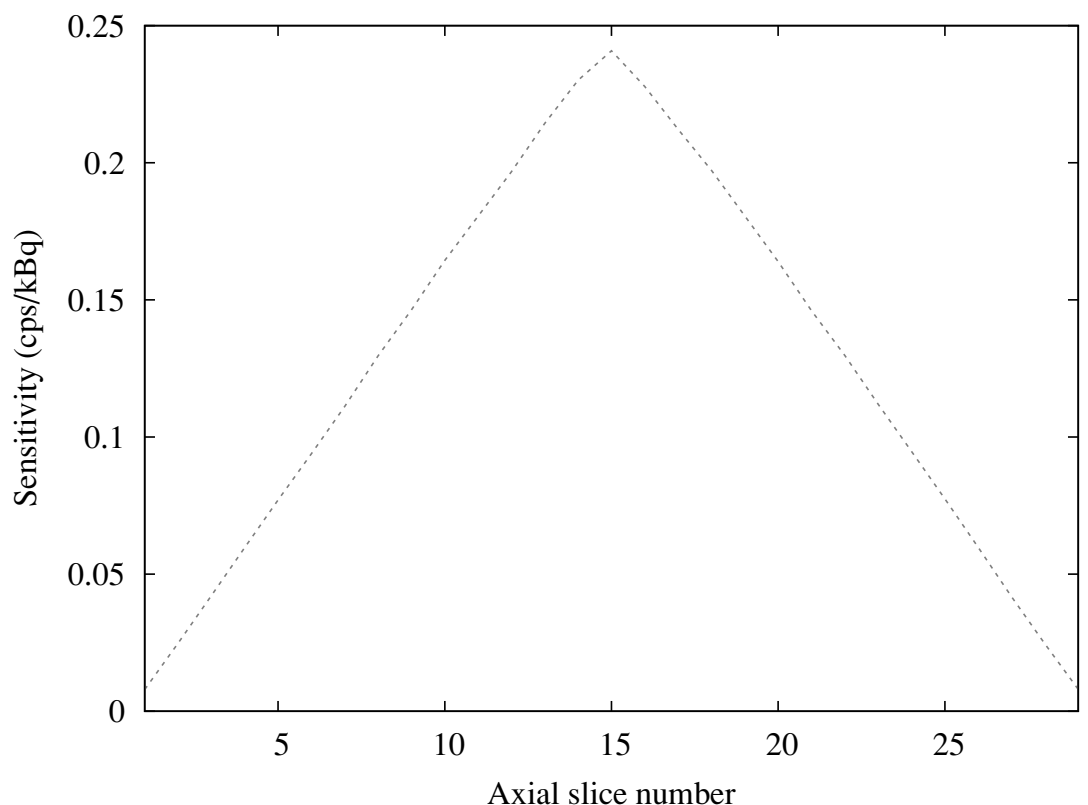

(c)

Figure 8. Sensitivity simulations: linear regression of the natural logarithm of the true count rates for (a) $0 \mathrm{~cm}$ radial offset, and (b) $10 \mathrm{~cm}$ radial offset. The sensitivity for true events (slice sensitivity profile) is shown in (c).

protocols for the assessment of PET scanners' performance, including spatial resolution, sensitivity and scatter fraction. On the other hand, the dead-time model was evaluated using the NEMA performance protocols for assessing a system's count rate characteristics. In addition to the standard NEMA NU2-2001 protocol performed for both the Allegro and GEMINI geometrical models, different phantom geometries were also simulated for the Allegro system model in order to assess the accuracy and potential limitations of the proposed dead-time model at the level of both singles and coincidences under different imaging conditions. Finally, the overall performance at the image level was evaluated using an image quality phantom based on the NEMA NU2-1994. 


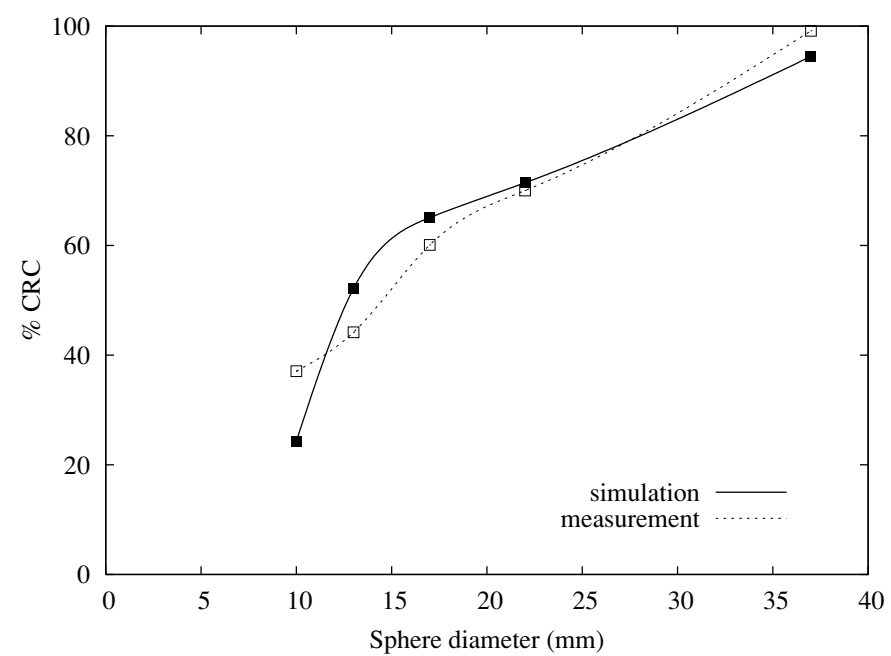

(a)

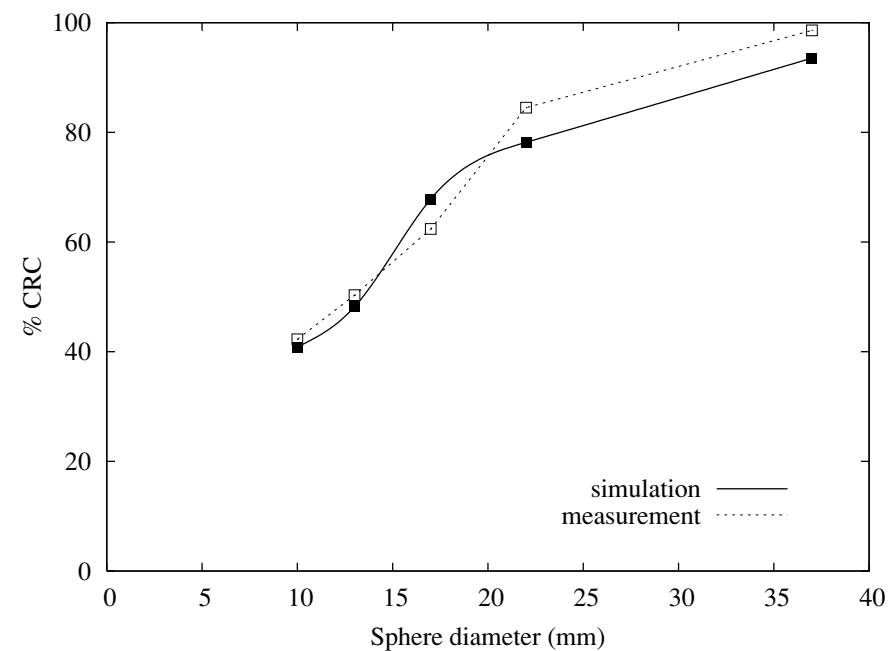

(b)

Figure 9. CRC $(\%)$ as a function of sphere size using reconstructed images obtained for measured and simulated datasets of the image quality phantom with a lesion to background activity ratio of (a) $4: 1$ and (b) 8:1.

In terms of spatial resolution, simulated results were consistently better in comparison to measurements irrespective of the direction considered. The degrading resolution in the axial direction, observed in measurements and already published data as a result of the use of FORE, were equally reproduced in the simulated results. The systematic difference observed between measured and simulated values can be potentially accounted by the absence of any modelling within GATE during the simulation of the detection process on effects such as light sharing between PMTs and block decoding. The level of underestimation will clearly vary depending on the block design and the sharing arrangement between crystal elements and photomultipliers. In the case of the Allegro the differences were between 11 and $18 \%$. The underestimation in the simulated resolution can be accounted for and modelled by introducing 
an analytical blurring to account for the light sharing effect (Bataille et al 2004). The same effects can also account for differences between the simulated and the measured sensitivity results which were $<10 \%$, with additional factors including the non-modelled PMT quantum efficiency as well as assuming uniform crystal efficiency throughout the scanner ring. Most importantly, no differences between simulated and measured sensitivity were seen as a function of position in the field of view, which may subsequently allow correcting for the overestimation of sensitivity in the simulated model by applying an appropriate global quantum efficiency factor in the crystals.

A high accuracy was observed in the scatter fraction provided by the Allegro and GEMINI models, found to vary between a minimum of $1 \%$ to a maximum of $3 \%$ of the measured scatter fraction, under substantially different imaging conditions. The developed dead-time model was assessed using count rate measurements performed considering the latest NEMA standards protocols and an activity range that encompasses the activity levels recommended and used in clinical whole body oncology and brain imaging studies. In addition, the activity levels used during the simulations were chosen to match the range available in already published measured results (Surti et al 2003, Surti 2004, Lodge et al 2004) used to validate the accuracy of the developed models. Signal flow effects not possible to model with GATE include pulse pile-up, the presence of overlapping triggering channels, electronics bandwidth limits and transfer to disk effects. Since no explicit modelling of pulse pile-up or overlapping triggering channels is possible within GATE, the accuracy of the dead-time model considered was assessed under variable imaging conditions using different NEMA phantoms simulating brain (NEMA NU2-1994) and whole body imaging (standard $20 \mathrm{~cm}$ diameter and a larger $35 \mathrm{~cm}$ diameter NEMA NU2-2001). Considering whole body imaging conditions, a good agreement throughout the range of activity concentrations considered was obtained for the true and random coincidences with a maximum difference of $<5 \%$ and $<8 \%$ respectively. Under all imaging conditions considered, a very good agreement was seen in the case of singles count rates irrespective of the activity concentration. Considering the geometry simulated by the NEMA NU2-1994 phantom, measured and simulated coincidence count rates were within 5\% for clinically relevant activity concentrations $\left(<25 \mathrm{kBq} \mathrm{ml}^{-1}\right)$, increasing to $<10 \%$ for activity concentrations corresponding to peak true count rates and total coincidences count rates of $>400 \mathrm{kcps}$. Differences between the accuracy of simulating true and random coincidences can be potentially accounted by the use of the same dead-time model for both types of coincidences, while this may not be the case in reality, particularly in the presence of a delayed coincidence window for the measurement of randoms. Finally, a combination of the different types of simulated coincidences lead to NEC rate curves exhibiting an equally good level of agreement with measurements, with a maximum of $8 \%$ differences at the peak NEC rates level considering all the different imaging conditions simulated.

The simulated singles count rate curves under imaging conditions leading to drastically different count rates do not exhibit obvious biases as a result of the absence of modelling pulse pile-up effects. Considering the activity levels likely to be encountered at clinical whole body and brain investigations, our proposed dead-time model has been shown to perform accurately with different simulated coincidence count rates within $5 \%$ of the measured rates. However, as already mentioned, the absence of modelling pulse pile-up effects may also lead to degradation in image quality through an associated degradation in spatial and energy resolutions. This was assessed using the image quality test for an activity concentration of $7.5 \mathrm{kBq} \mathrm{ml}^{-1}$ (corresponding to a singles count rate of 5-6 kcps for the given phantom geometry). This image quality test did not reveal any contrast degradation that could be caused by event mis-positioning due to pulse pile-up effects which are not explicitly modelled. This could be accounted by the local centroid algorithms used on PMT signals to localize the 
detected events. On the other hand, these results do not exclude that at higher count rates (considering, for example, different phantom geometries including activities outside the field of view) these parameters do become significant and may introduce larger errors in simulated count rates and associated image contrast. The developed dead-time model does not explicitly model all of the individual electronic processes taking place during an event readout and as such remains an approximation to the real signal flow. Therefore, although shown to be valid for clinically relevant activity levels under different imaging conditions, it is not destined for testing the performance of new detector electronic readout components or upgrades.

Finally, the overall performance of the developed model was tested in terms of reconstructed image quality considering activity levels within the range of simulated activity levels used in the count rate performance validation. The use of the same image reconstruction and correction algorithms for the simulated datasets as for the measured datasets allowed the unbiased evaluation of simulated image quality. A close agreement between simulated and measured contrast recovery coefficients was obtained for two different target-to-background ratios likely to be encountered in clinical practice considering a number of different sphere sizes ranging in volume from $0.5 \mathrm{ml}$ to $27 \mathrm{ml}$. In addition, the good agreement established on the background signal-to-noise ratio provides further proof supporting the accuracy of the overall developed simulation model under clinical imaging conditions.

\section{Conclusions}

We have validated the use of GATE, a newly developed Monte Carlo code for the simulation of PET and SPECT imaging systems, by demonstrating the feasibility and accuracy of performing a comprehensive simulation of the Phillips Allegro/GEMINI PET scanners. The incorporation of electronics dead-time modelling has also been described and validated against experimental measurements. The good agreement between simulated and measured results supports the accuracy of the developed geometry and signal flow models for a large range of activity concentrations encompassing those in the range likely to be encountered in clinical practice. The developed Philips PET scanners model with GATE can be used in the optimization of emission acquisition protocols, and validation of reconstruction and data correction algorithms.

\section{Acknowledgments}

The authors would like to thank all the developers of GATE for their hard work and their assistance. We would also like to thank Suleman Surti and Martin Lodge for providing us with some of the measured data used in the validation studies during this work. One of us (DV) would like to thank Ramsey Badawi, during the revision of this manuscript, for useful discussions on the potential effects of pulse pile-up in performance characterization of PET systems. We are also grateful to Daniel Gagnon of Philips Medical Systems for his guidance during the design of our model and Thomas Frach, Klaus Fiedler, Henning Braess and Marc Bush of Philips Medical Systems for their help with decoding the Philips sinogram format. Frederic Lamare is supported by a thesis grant from Philips Medical Systems. This work was supported by a Region of Brittany grant (programme 1044: QUANTEP).

\section{References}

Agostinelli S et al 2003 GEANT4 - a simulation toolkit Nucl. Instrum. Methods A 506 250-303

Allegro User's Manual 2002 Philips 
Bataille F et al 2004 Monte Carlo simulation for the ECAT HRRT using GATE IEEE Medical Imaging Conference Proceedings

Bielajew A F et al 1994 History, overview and recent improvements of EGS4 NRCC Report PIRS-0436, National Research Council Ottawa, Canada

Brasse D et al 2005 Correction methods for random coincidences in fully 3D whole body PET: impact on data and image quality J. Nucl. Med. 46 859-67

Briesmeister J F (ed) 1993 MCNP-a general Monte Carlo N-particle transport code LANL Report LA-12625-M, Los Alamos National Laboratory, Los Alamos, NM, USA

Brun R and Rademakers F 1997 ROOT-an object oriented data analysis framework Nucl. Instrum. Methods A 389 81-6

Buvat I and Castiglioni I 2002 Monte Carlo simulations in SPECT and PET Q. J. Nucl. Med. 46 48-61

Daube-Witherspoon M E et al 2002 PET performance measurements using the NU2-2001 standard J. Nucl. Med. 43 1398-409

Jan S et al 2004 GATE: a simulation toolkit for PET and SPECT Phys. Med. Biol. 49 4543-61

Karp J S, Muehllehner G, Neerbohm D and Mankoff D A 1996 Event localization in a continuous scintillation detector using digital processing IEEE Trans. Nucl. Sci. 33 550-5

Knoll G F 2000 Radiation Detection and Measurement 3rd edn (New York: Wiley)

Kolthammer J 2004 personal communication, Philips Medical Systems, Cleveland, OH

Lodge M A 2004 personal communication

Lodge M A, Dilsizian V and Line B R 2004 Performance assessment of the Philips GEMINI PET/CT scanner J. Nucl. Med. $\mathbf{4 5} 425$

National Electrical Manufacturers Association 1994 NEMA Standards Publication NU2-1994: Performance Measurements of Positron Emission Tomographs (Washington DC: NEMA)

National Electrical Manufacturers Association 2001 NEMA Standards Publication NU2-2001: Performance Measurements of Positron Emission Tomographs (Washington DC: NEMA)

Smith R J et al 1997 A comparison of segmentation and emission subtraction for singles transmission in PET IEEE Trans. Nucl. Sci. 45 1212-8

Strother S C, Casey M E and Hoffman E J 1990 Measuring PET scanner sensitivity: relating count rate to image signal to noise ratios using noise equivalent counts IEEE Trans. Nucl. Sci. 37 783-8

Surti S et al 2003 A multi-scanner evaluation of PET image quality using phantom studies Conf. Rec. IEEE Nucl. Sci. Symp. Med. Imag. Conf. (Portland, OR)

Surti S 2004 personal communication

Surti S and Karp J S 2004 Imaging characteristics of a 3-dimensional GSO whole-body PET camera J. Nucl. Med. 45 1040-9

Talguen V et al 2004 Evaluation of attenuation correction methodology in the Allegro PET system IEEE Trans. Nucl. Sci. 51 2688-92

Visvikis D et al 2004 Characterisation of SUV accuracy in FDG PET using 3D RAMLA and the Philips Allegro PET scanner J. Nucl. Med. 45103 